\begin{tabular}{|c|c|}
\hline 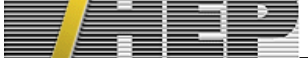 & 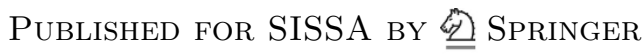 \\
\hline & $\begin{array}{l}\text { RECEIVED: April 1, } 2016 \\
\text { ACCEPTED: April 29, } 2016 \\
\text { Published: May 13, } 2016\end{array}$ \\
\hline
\end{tabular}

\title{
Search for sphalerons: IceCube vs. LHC
}

\author{
John Ellis, ${ }^{a, b}$ Kazuki Sakurai ${ }^{c}$ and Michael Spannowsky ${ }^{c}$ \\ ${ }^{a}$ Theoretical Particle Physics and Cosmology Group, Physics Department, King's College London, \\ London WC2R 2LS, U.K. \\ ${ }^{b}$ Theoretical Physics Department, CERN, \\ CH-1211 Geneva 23, Switzerland \\ ${ }^{c}$ Institute for Particle Physics Phenomenology, Department of Physics, University of Durham, \\ Science Laboratories, South Road, Durham, DH1 3LE, U.K. \\ E-mail: John.Ellis@cern.ch, kazuki.sakurai@durham.ac.uk, \\ michael.spannowsky@durham.ac.uk
}

ABSTRACT: We discuss the observability of neutrino-induced sphaleron transitions in the IceCube detector, encouraged by a recent paper by Tye and Wong (TW), which argued on the basis of a Bloch wave function in the periodic sphaleron potential that such transitions should be enhanced compared to most previous calculations. We calculate the dependence on neutrino energy of the sphaleron transition rate, comparing it to that for conventional neutrino interactions, and we discuss the observability of tau and multi-muon production in sphaleron-induced transitions. We use IceCube 4-year data to constrain the sphaleron rate, finding that it is comparable to the upper limit inferred previously from a recast of an ATLAS search for microscopic black holes at the LHC with $\sim 3 / \mathrm{fb}$ of collisions at $13 \mathrm{TeV}$. The IceCube constraint is stronger for a sphaleron barrier height $E_{\mathrm{Sph}} \gtrsim 9 \mathrm{TeV}$, and would be comparable with the prospective LHC sensitivity with $300 / \mathrm{fb}$ of data at $14 \mathrm{TeV}$ if $E_{\mathrm{Sph}} \sim 11 \mathrm{TeV}$.

Keywords: Neutrino Physics, Nonperturbative Effects, Scattering Amplitudes

ARXIV EPRINT: 1603.06573 


\section{Contents}

1 Introduction 1

2 Neutrino-nucleon cross-section calculations 2

3 Leptons in sphaleron-induced transitions 5

$\begin{array}{lll}4 & \text { IceCube constraints on sphaleron-induced transitions } & 7\end{array}$

5 Summary and conclusions $\quad 9$

\section{Introduction}

The recent discovery of the Higgs boson with a mass of $125 \mathrm{GeV}$ by ATLAS and CMS $[1,2]$ completes the electroweak sector of the Standard Model. Ongoing measurements of interactions of Higgs and gauge bosons find good agreement with perturbative predictions of the Standard Model with its spontaneously broken $\mathrm{SU}(2) \times \mathrm{U}(1)$ gauge group [3-8]. A direct consequence of the $\mathrm{SU}(2)$ gauge group, beyond the perturbative regime, is the existence of non-perturbative topological effects. Field configurations with finite Euclidean action are classified by an integer topological winding number, namely the Chern-Simons number $N_{\mathrm{CS}}$. Topologically distinct ground states are separated by an energy barrier, and the sphaleron [9] is an extremal saddle point on top of the barrier with half-integer $N_{\mathrm{CS}}$ and an energy $E_{\mathrm{Sph}} \simeq 9 \mathrm{TeV}$. Owing to the Adler-Bell-Jackiw anomaly, transitions through sphaleron configurations would lead to striking $(\mathrm{B}+\mathrm{L})$-violating processes. Direct observation of such interactions can be of capital importance in explaining the mechanism underlying the cosmological baryon asymmetry [10-14], which might arise from the transmutation of a primordial lepton asymmetry.

While the energy of the sphaleron is directly linked to the shape of the effective potential for the Chern-Simons number and is thus rather undisputed, its production rate is subject to large theoretical uncertainties [15-18]. Over the years, there have been many estimates of the rate of sphaleron transitions in high-energy collisions, most of them with discouraging results for the prospects for experimental searches. However, a new approach [9], exploiting the periodicity of the Chern-Simons potential, has challenged this paradigm and argued in favour of enhanced sphaleron-induced transition rates.

The authors indicate that, since the effective Chern-Simons potential is periodic, it is appropriate to use a Bloch wave function to estimate the sphaleron-induced transition rate. They find that, although the transition rate is still strongly suppressed at energies below the sphaleron threshold energy, the rate may not be suppressed at higher energies above $E_{\mathrm{Sph}}$.

Although the new analysis of [9] is not universally accepted by the community, and further theoretical scrutiny is needed, it motivated two of us (JE and KS) to analyze the 
observability of sphaleron-induced transitions at the LHC and possible future higher-energy $p p$ colliders, and recast a recent ATLAS search for microscopic black holes using $\sim 3 / \mathrm{fb}$ of data at $13 \mathrm{TeV}$ in the centre-of-mass as a search for sphalerons [19]. In this way, expanding on previous proposals $[20,21]$, we were able to establish for the first time a significant direct experimental constraint on sphaleron-induced transitions, assess the potential improvement in sensitivity of future LHC runs with higher integrated luminosities and possibly energy, and preview the corresponding prospects for possible future higher-energy $p p$ colliders.

In this connection, it is important to remember that cosmic rays provide collisions with centre-of-mass energies beyond those attainable with the LHC. However, the low fraction of ultra-high-energy $p p$ collisions that would produce sphaleron transitions, combination with the limitations of the Auger experiment [22] for extracting detailed information from air showers renders challenging this avenue in searches for sphalerons [23].

Better prospects may be offered by ultra-high-energy neutrino events [24-28], which could be observed in a cubic-kilometer neutrino telescopes, such as IceCube $[29,30]$ and KM3NeT [31]. As we discuss in more detail below, the estimates of TW suggest that the rate of sphaleron-induced transitions in the highest-energy cosmic-ray collisions observed by IceCube could be comparable to the conventional neutrino cross section, and might even dominate the interactions of cosmogenic neutrinos [32] produced by the Greisen-ZatsepinKuzmin (GZK) process [33, 34]: $p+\gamma_{\mathrm{CMB}} \rightarrow \pi^{+} \rightarrow \nu$.

Accordingly, in this paper we extend the analysis of ES to ultra-high-energy neutrino interactions, estimating the upper limit on sphaleron transitions obtainable from present IceCube results and considering the implications for GZK neutrinos. Remarkably, we find that the present IceCube sensitivity is very similar to that of the first LHC data at $13 \mathrm{TeV}$. If the rate of sphaleron transitions were to saturate the present LHC limit, the rate of GZK neutrino interactions would be significantly higher than is conventionally estimated, improving significantly the prospects for their future detection with IceCube or a cubickilometre detector.

\section{Neutrino-nucleon cross-section calculations}

The analysis of [9] is based on the idea that sphaleron transitions changing the ChernSimons number $n$ can be modelled by considering a Bloch wave function for an effective one-dimensional Schrödinger equation

$$
\left(-\frac{1}{2 m} \frac{\partial^{2}}{\partial Q^{2}}+V(Q)\right) \Psi(Q)=E \Psi(Q),
$$

where $m$ is an effective "mass" parameter variously estimated to be $\sim 17.1 \mathrm{TeV}[35]$ to $\sim 22.5 \mathrm{TeV}[36]$ and the effective potential is taken from [35]:

$$
V(Q) \simeq 4.75\left(1.31 \sin ^{2}\left(Q m_{W}\right)+0.60 \sin ^{4}\left(Q m_{W}\right)\right) \mathrm{TeV} .
$$

The sphaleron barrier height $E_{\mathrm{Sph}}$ is the maximum value of the effective potential $V(Q)$, which is $E_{\mathrm{Sph}}=9.11 \mathrm{TeV}$ in a pure $\mathrm{SU}(2)$ theory, and is estimated to be reduced by $\sim 1 \%$ 
when the U(1) of the Standard Model is included. Following [9], we assume $E_{\mathrm{Sph}}=9 \mathrm{TeV}$ as a nominal value, but present some numerical results for $E_{\mathrm{Sph}} \in[8,11] \mathrm{TeV}$.

Ref. [9] found that the pass-band structure in the Bloch wave function approach reproduced the expected (near-exponential) tunnelling suppression of sphaleron transitions in collisions of quark partons with subprocess centre-of-mass energies $\sqrt{\hat{s}} \ll E_{\mathrm{Sph}}$, but found that there is no suppression for $\sqrt{\hat{s}} \geq E_{\mathrm{Sph}}$. They found that the rates of sphaleron-induced transitions were similar for the two values of $m$, and adopted $m=17.1 \mathrm{TeV}$ for definiteness. The result of the TW analysis can be expressed as partonic cross-section

$$
\hat{\sigma}(\Delta n= \pm 1) \propto \exp \left(c \frac{4 \pi}{\alpha_{W}} S(\sqrt{\hat{s}})\right)
$$

where $c \sim 2$ and the suppression factor $S(\sqrt{\hat{s}})$ is shown in figure 8 of [9]. As discussed in [19], we approximate $S(\sqrt{\hat{s}})$ at intermediate energies by

$$
S(\sqrt{\hat{s}})=(1-a) \overline{\sqrt{\hat{s}}}+a \overline{\hat{s}}-1 \quad \text { for } \quad 0 \leq \overline{\sqrt{\hat{s}}} \leq 1
$$

where $\overline{\sqrt{\hat{s}}} \equiv \sqrt{\hat{s}} / E_{\mathrm{Sph}}$ and $a=-0.005$.

In the absence of a reliable calculation of the overall magnitude of eq. (2.3), following [19] we parametrize the partonic cross section for the sphaleron-induced neutrino-quark collision as

$$
\hat{\sigma}_{q \nu}(\hat{s})=\frac{p}{m_{W}^{2}},
$$

for $\sqrt{\hat{s}}>E_{\mathrm{Sph}}$ and apply the suppression factor (2.4) for $\sqrt{\hat{s}}<E_{\mathrm{Sph}}$. Our numerical results are relatively insensitive to the form of this suppression factor. The overall factor $p$ in (2.5) depends in general on $\hat{s}$ [9]. However, our result is also not very sensitive to such an energy dependence, since (as we discuss below) the interaction is dominated by subprocess energies near the threshold $\sqrt{\hat{s}} \gtrsim E_{\mathrm{Sph}}$, due to the sharply-falling cosmogenic neutrino flux. The cross section for sphaleron transitions in neutrino-nucleon collisions is given by

$$
\sigma_{\nu N}\left(E_{\nu}\right)=\sum_{q} \int_{0}^{1} d x f_{q}(x, \mu) \hat{\sigma}_{q \nu}\left(2 x m_{N} E_{\nu}\right),
$$

where $f_{q}(x, \mu)$ is a parton distribution function for the quark flavour $q$ and $m_{N}$ is the mass of nucleon. The neutrino-nucleon centre-of-mass energy $\hat{E}=\sqrt{2 m_{N} E_{\nu}}$, neglecting the $m_{N}^{2}$ term, and the neutrino-parton subprocess centre-of-mass energy $\sqrt{\hat{s}}=\sqrt{2 x m_{N} E_{\nu}}$.

Figure 1 displays the energy dependence of the cross section for sphaleron-induced transitions calculated under these assumptions with $c=2$ and $p=1$ : the results are insensitive to $c \in[1,4]$ and scale linearly with $p$. The solid (dot-dashed) (dashed) red lines are for $E_{\mathrm{Sph}}=9(8)(10) \mathrm{TeV}$, and the black dashed line is the sum of the conventional charged- and neutral-current neutrino cross sections. We see that the sphaleron-induced cross section would dominate for $E_{\nu} \gtrsim 2 \times 10^{8} \mathrm{TeV}$ if $p=1$, but recall that this factor is quite uncertain.

The cross section estimates in figure 1 can be convoluted with the cosmogenic neutrino flux, $d^{2} \Phi /\left(d E_{\nu} d t d \Omega\right)\left[\mathrm{GeV}^{-1} \mathrm{~cm}^{-2} \mathrm{~s}^{-1} \mathrm{sr}^{-1}\right]$, to calculate the event rates. We use the cosmogenic neutrino flux estimated in [37] throughout this paper. While we use the central 


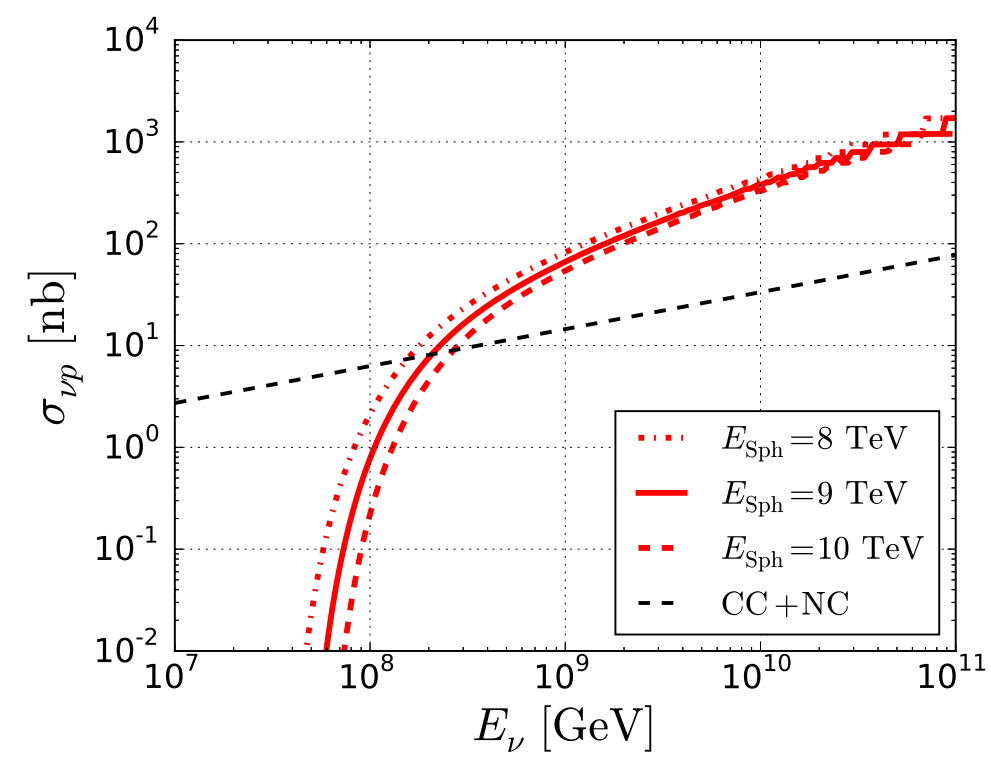

Figure 1. Compared to the sum of the conventional charged- and neutral-current neutrino cross sections (black dashed line), we show the energy dependence of the cross section for sphaleron transitions in neutrino collisions for a barrier height $E_{\mathrm{Sph}}=9 \mathrm{TeV}, c=2$ and $p=1$ in eq. (2.5) with $S$ given by (2.4) (red solid curve) and, for comparison, choices $E_{\mathrm{Sph}}=8$ and $10 \mathrm{TeV}$ (red dot-dashed and dashed lines, respectively). The variations in the sphaleron curves for $1 \leq c \leq 4$ are within the widths of the lines, but we recall that the overall normalization factor $p$ is quite uncertain.

value of their estimate, we note that this choice is subject to uncertainties. The event rate in the IceCube detector also depends on the energy-dependent effective neutrino detection area, $A_{\text {eff }}\left(E_{\nu}\right)$, which has been evaluated by the IceCube collaboration [38] using conventional neutrino-nucleon interaction. Assuming the same detection efficiency, we estimate the sphaleron-induced IceCube event rate as

$$
\frac{d N_{\mathrm{Sph}}}{d t}=\int_{E_{\nu}^{\text {thres }}} d E_{\nu} \int d \Omega \frac{\sigma_{\nu N}^{\mathrm{Sph}}\left(E_{\nu}\right)}{\sigma_{\nu N}^{\mathrm{CC} / \mathrm{NC}}\left(E_{\nu}\right)} A_{\mathrm{eff}}\left(E_{\nu}\right) \frac{d^{2} \Phi}{d E_{\nu} d t d \Omega},
$$

where $E_{\nu}^{\text {thres }}$ is the energy threshold of incoming cosmogenic neutrinos. In the second integral we take into account only neutrinos coming from the upper hemisphere of IceCube, since the neutrinos from the lower hemisphere will be absorbed by the interaction with the Earth. In figure 2 we show the sphaleron-induced and conventional IceCube event rate as functions of $E_{\nu}^{\text {thres }}$ again assuming $c=2$ and $p=1$ and using (dot-dashed) (dashed) red lines for $E_{\mathrm{Sph}}=9(8)(10) \mathrm{TeV}$ and a black dashed line for sum of the conventional charged- and neutral-current neutrino cross sections. We see that the sphaleron-induced transitions would dominate over conventional neutrino collisions by a factor $\gtrsim 5$ for all $E_{\nu}^{\text {thres }} \geq 10^{7} \mathrm{GeV}$ if $p=1$.

Figure 3 displays some characteristics of the sphaleron-induced transitions. In the left panel we show a breakdown of the collision rates with respect to the quark parton species 


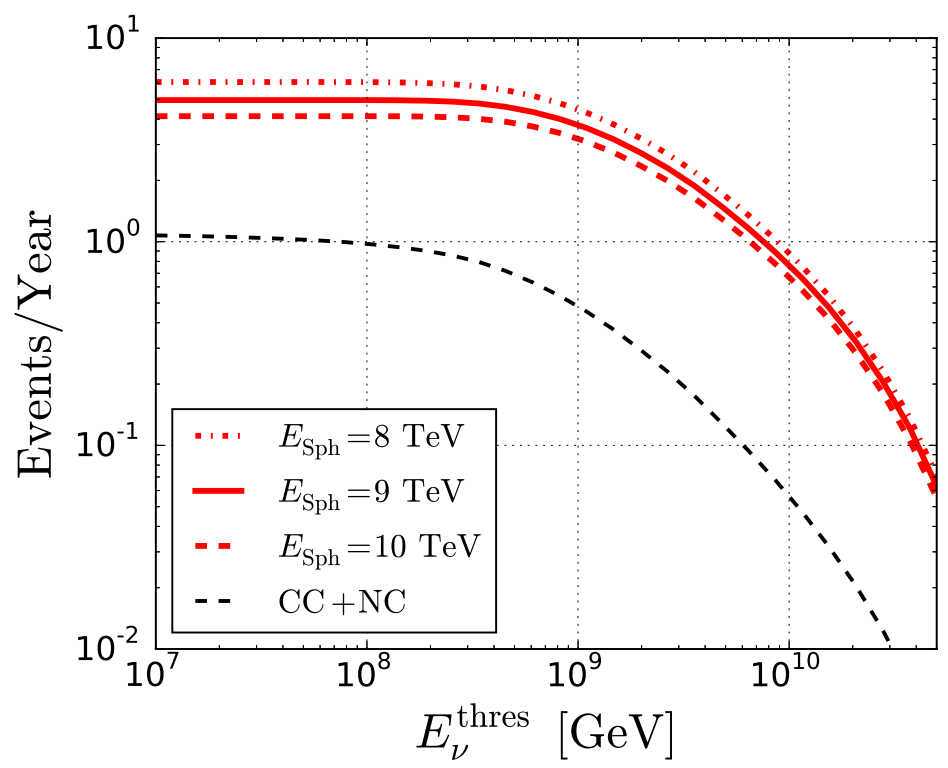

Figure 2. Compared to the sum of the conventional charged- and neutral-current neutrino cross sections (black dashed line), we show the rate for sphaleron transitions in IceCube for a barrier height $E_{\mathrm{Sph}}=9 \mathrm{TeV}, p=1$ in eq. (2.5) (red solid curve) and, for comparison, choices $E_{\mathrm{Sph}}=8$ and $10 \mathrm{TeV}$ (red dot-dashed and dashed lines, respectively).

inside the nucleon targets in the ice. As was to be expected, interactions with $u$ and $d$ quarks dominate, followed by interactions with antiquarks and heavy flavours. In the right panel we show the corresponding distributions in the reduced neutrino-quark subprocess centre-of-mass energies $\sqrt{\hat{s}}$, which are sharply peaked at the sphaleron energy $E_{\mathrm{Sph}}$, taken here to have its nominal value of $9 \mathrm{TeV}$. This peaking implies that our results would not be affected strongly by a possible energy dependence in the overall factor $p$, but depend essentially only on the value of $p$ at the sphaleron threshold energy.

\section{Leptons in sphaleron-induced transitions}

In the IceCube detector [30], neutral current interaction and charged current interaction of electron neutrinos leave a shower-like signature, whilst high energy muons and very high energy taus $\left(E_{\tau}>10^{7} \mathrm{GeV}\right)$ leave a track-like signature. IceCube expects to be able to see a 'double-bang' signature for $\tau$ leptons with energies $\in\left[10^{6}, 10^{7}\right] \mathrm{GeV}$.

We simulate distributions of leptons $(\mu$ and $\tau$ ) produced by the sphaleron-induced neutrino-quark collision events in parton level. We consider the simplest possibility of such events: $q \nu \rightarrow 8 \bar{q} 2 \bar{\ell}$ induced by the gauge invariant $(\bar{q} \bar{q})_{1}(\bar{q} \bar{q} \bar{q})_{2}(\bar{q} \bar{q} \bar{q})_{3}\left(\bar{\ell}_{1} \bar{\ell}_{2} \bar{\ell}_{3}\right)$ operator, where the suffix denotes the generation. We assume equal flux for each flavour of cosmogenic neutrinos. Leptons can be produced either directly from the primary interaction, $q \nu \rightarrow 8 \bar{q} 2 \bar{\ell}$, or secondarily from the decay of the heavy particles $(t$ and $W)$. 

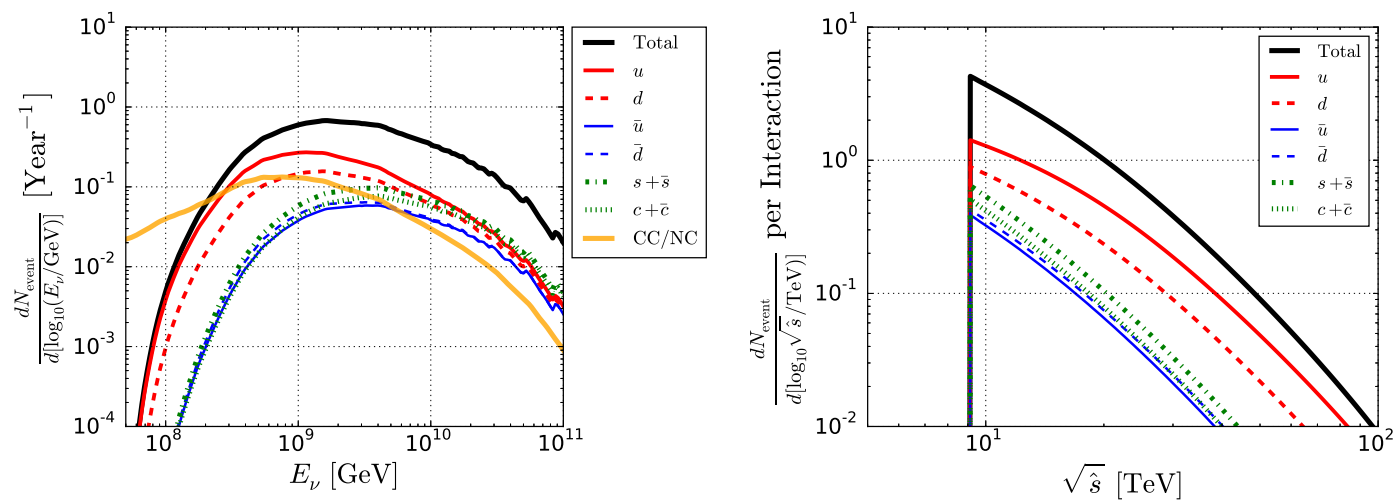

Figure 3. Left panel: contributions to the total cross section for sphaleron transitions in neutrino collisions in IceCube, for the nominal case $E_{\mathrm{Sph}}=9 \mathrm{TeV}$ and $p=1$ in (2.5) with $S$ given by (2.4). The solid green curve is for the sum of conventional charged- and neutral-current neutrino interactions. Right panel: the distributions in the neutrino-parton reduced centre-of-mass energy, which is peaked at $E_{\mathrm{Sph}}$. In both panels, the contributions of different parton-parton collision processes are colour-coded as indicated.
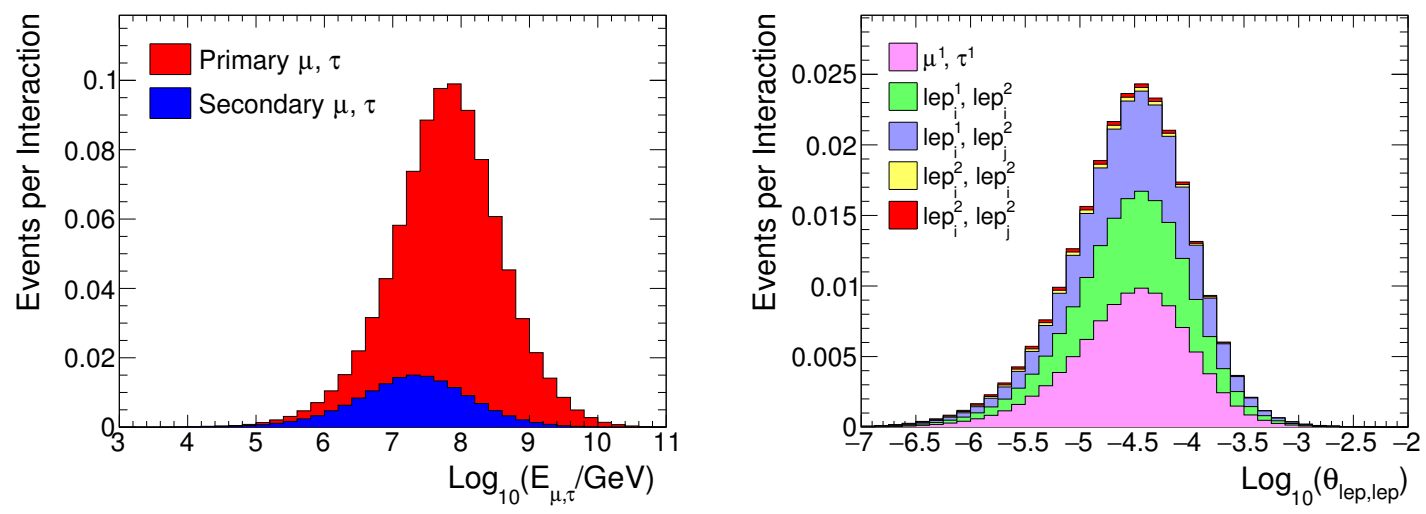

Figure 4. Left panel: histograms of the primary and secondary muon energy distributions (red and blue, respectively) in sphaleron-induced transitions in neutrino-nucleon collisions in IceCube for $E_{\mathrm{Sph}}=9 \mathrm{TeV}$, normalised to a single sphaleron-induced event. Right panel: histograms of the opening angles in the laboratory frame between pairs of leptons in sphaleron transitions in neutrino interactions in IceCube for $E_{\mathrm{Sph}}=9 \mathrm{TeV}$, colour-coded for the different combinations of primary and secondary leptons.

The left panel of figure 4 displays the primary and secondary $\mu$ and $\tau$ energy distributions (which are identical) normalised to a single sphaleron-induced event. We see that the primary lepton energies are peaked just below $10^{8} \mathrm{GeV}$, whereas the secondary lepton energies are peaked closer to $10^{7} \mathrm{GeV}$. IceCube expects to be able to see a 'double-bang' signature for $\tau$ leptons with energies $\in\left[10^{6}, 10^{7}\right] \mathrm{GeV}$. We see that sphaleron-induced transitions would produce some primary and secondary $\tau$ leptons in this energy range. However, we find only $5 \%$ of the sphaleron-induced events have $\tau$ leptons in this energy range. 
Since the sphaleron-induced interaction can produce multiple leptons, IceCube might be able to see multiple tracks in the event if those tracks are separated more than $17 \mathrm{~m}$. This separation typically requires the opening angle of the leptons to be $\gtrsim 2 \cdot 10^{-2}$. The right panel of figure 4 displays histograms of the $\ell-\ell$ opening angles in the laboratory frame, colour-coded for the different combinations of primary and secondary leptons, and again normalised to a single sphaleron-induced event. We see that the opening angles are in all cases much smaller than the IceCube angular resolution, so we do not expect multiple lepton tracks to be distinguished.

\section{IceCube constraints on sphaleron-induced transitions}

In the absence of a distinctive leptonic signature, we use the generic IceCube search for which detection efficiency is encoded in the effective neutrino detection area given in [38]. Moreover, we assume in the absence of a detailed simulation of the IceCube efficiency for detecting sphaleron-induced final states that it is the same as that for conventional final states, and that the neutrino spectrum keeps falling at energies above $10^{11} \mathrm{GeV}$. Figure 5 compares the upper limit on the overall cross-section factor $p$ obtained in this way from IceCube 4-year [39] (solid red lines) with the upper limits derived in [19] from recasting the ATLAS Run 2 search for microscopic black holes with $\sim 3 / \mathrm{fb}$ of data at $13 \mathrm{TeV}$ [40] (solid blue lines). The upper panel of figure 5 is for $\Delta n=-1$ transitions, which yield final states with 10 energetic particles at the LHC, and the lower panel is for $\Delta n=+1$ transitions, which yield 14-particle final states at the LHC.

The LHC constraints in figure 5 are different for the 10- and 14-particle final states, and quite sensitive to the assumed value of the sphaleron energy $E_{\mathrm{Sph}}$. (We recall that our nominal value is $E_{\mathrm{Sph}}=9 \mathrm{TeV}$, but we display results for $E_{\mathrm{Sph}} \in[8,11] \mathrm{TeV}$.) This is because the rate for sphaleron-induced transitions at the LHC with a centre-of-mass energy of $13 \mathrm{TeV}$ is quite sensitive to $E_{\mathrm{Sph}}$. In contrast, the IceCube 4-year limit is quite insensitive to $E_{\mathrm{Sph}}$ over the range studied, because of the larger range of neutrino energies. Within our assumptions, the IceCube efficiencies and hence limits for $\Delta n= \pm 1$ transitions are the same, whereas the LHC limits are stronger for $\Delta n=+1$ transitions, for which we estimated in [19] a greater detection efficiency.

By a remarkable coincidence, we see that the LHC and IceCube constraints are almost identical for the nominal value $E_{\mathrm{Sph}}=9 \mathrm{TeV}$, but the IceCube limits are stronger for larger $E_{\mathrm{Sph}}$, becoming some 3 orders of magnitude stronger for $E_{\mathrm{Sph}}=11 \mathrm{TeV}$.

We also display in figure 5 the prospective future LHC exclusion sensitivities for higher integrated luminosities (dashed and dot-dashed blue lines) and energy (dashed and dotdashed green lines). We see that $300 / \mathrm{fb}$ of luminosity at $14 \mathrm{TeV}$ would be needed for the LHC sensitivity to surpass the IceCube constraint for $E_{\mathrm{Sph}}=11 \mathrm{TeV}$. We anticipate that the IceCube sensitvity will also be improved by longer operating time and/or effective size, and note that an order-of-magnitude improvement in the IceCube sensitivity would make it highly competitive with the LHC with $3000 / \mathrm{fb}$ for $E_{\mathrm{Sph}}=11 \mathrm{TeV}$, with both being able to reach $p \simeq 10^{-2}$. On the other hand, for the nominal value $E_{\mathrm{Sph}}=9 \mathrm{TeV}$, the $\mathrm{LHC}$ would have a greater reach than IceCube, down to $p<10^{-4}$. For $p \ll 10^{-1}$, the sphaleron 

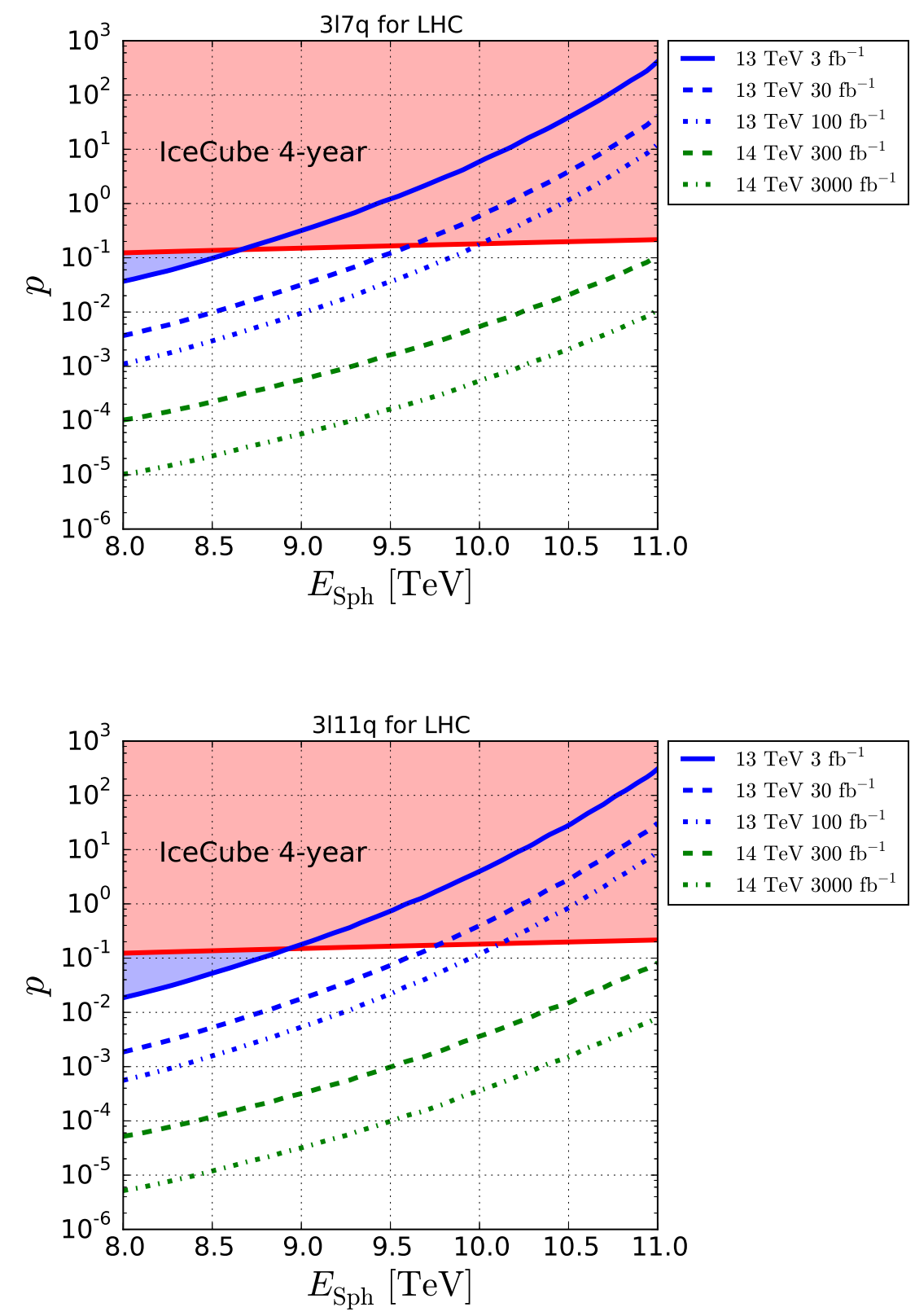

Figure 5. Comparisons of the constraint obtained from IceCube 4-year data [39] (near-horizontal red solid line) with that obtained from a recast of the ATLAS search for microscopic black holes with $\sim 3 / \mathrm{fb}$ of collisions at $13 \mathrm{TeV}$ (solid blue line). Also shown are prospective LHC sensitivities with increased luminosity and/or centre-of-mass energy. The comparisons are for $8 \mathrm{TeV} \leq E_{\mathrm{Sph}} \leq$ $10 \mathrm{TeV}$ for $\Delta n=-1$ sphaleron transitions (upper panel) and $\Delta n=+1$ transitions (lower panel). 
cross-section at IceCube becomes smaller than the cross-section for conventional neutrino interactions, and searching for sphaleron events using the IceCube event rate alone becomes difficult. A detailed study how to discriminate sphaleron events from conventional neutrino events would be required in this case.

\section{Summary and conclusions}

We have shown that IceCube could have a sensitivity to spahaleron-induced transitions that is comparable to that of the LHC. For a cross-section prefactor $p=1$, the rate of such transitions in neutrino collisions would exceed the sum of conventional charged- and neutral-current interactions for $E_{\nu} \gtrsim 2 \times 10^{8} \mathrm{GeV}$, as seen in figure 1, yielding a larger number of events for a neutrino threshold energy above $E_{\nu}=10^{7} \mathrm{GeV}$, as seen in figure 2 . Our simulations of neutrino-induced sphaleron transitions do not reveal any distinctive leptonic signatures, with a limited fraction of 'double-bang' $\tau$ events, as seen in the left panel of figure 4, and multilepton bundles that are probably not resolvable, as seen in the right panel of figure 4 .

Remarkably, the prospective IceCube constraints on sphaleron-induced transitions are comparable to those from the LHC, as seen in figure 5, with IceCube having an advantage for large sphaleron energies $E_{\mathrm{Sph}}$ and the LHC at small $E_{\mathrm{Sph}}$. The crossover is currently close to the nominal value $E_{\mathrm{Sph}}=9 \mathrm{TeV}$.

Our estimates need to be validated by dedicated experimental simulations for IceCube as well as for the LHC, but our results indicate that both have interesting sensitivities for sphaleron-induced transitions, able to probe significantly below $p=1$, and hence able to test or constrain the suggestion by Tye and Wong [9] that sphaleron transitions may be much less suppressed than commonly thought previously.

\section{Acknowledgments}

The work of JE was supported partly by the London Centre for Terauniverse Studies (LCTS), using funding from the European Research Council via the Advanced Investigator Grant 26732, and partly by the STFC Grant ST/L000326/1. He thanks Henry Tye, Sam Wong and Andy Cohen for instructive discussions. KS and MS are supported by STFC through the IPPP grant.

Open Access. This article is distributed under the terms of the Creative Commons Attribution License (CC-BY 4.0), which permits any use, distribution and reproduction in any medium, provided the original author(s) and source are credited.

\section{References}

[1] ATLAS collaboration, Observation of a new particle in the search for the standard model Higgs boson with the ATLAS detector at the LHC, Phys. Lett. B 716 (2012) 1 [arXiv: 1207.7214] [INSPIRE]. 
[2] CMS collaboration, Observation of a new boson at a mass of $125 \mathrm{GeV}$ with the CMS experiment at the LHC, Phys. Lett. B 716 (2012) 30 [arXiv:1207.7235] [INSPIRE].

[3] DELPHI, OPAL, LEP Electroweak, ALEPH and L3 collaborations, S. Schael et al., Electroweak measurements in electron-positron collisions at $W$-boson-pair energies at LEP, Phys. Rept. 532 (2013) 119 [arXiv:1302.3415] [INSPIRE].

[4] ATLAS and CMS collaborations, Measurements of the Higgs boson production and decay rates and constraints on its couplings from a combined ATLAS and CMS analysis of the LHC pp collision data at $\sqrt{s}=7$ and $8 \mathrm{TeV}$, ATLAS-CONF-2015-044, CERN, Geneva Switzerland (2015).

[5] ATLAS collaboration, Measurement of $W^{+} W^{-}$production in pp collisions at $\sqrt{s}=7 \mathrm{TeV}$ with the ATLAS detector and limits on anomalous $W W Z$ and $W W \gamma$ couplings, Phys. Rev. D 87 (2013) 112001 [arXiv:1210.2979] [INSPIRE].

[6] ATLAS collaboration, Measurement of $Z Z$ production in pp collisions at $\sqrt{s}=7$ TeV and limits on anomalous $Z Z Z$ and $Z Z \gamma$ couplings with the ATLAS detector, JHEP 03 (2013) 128 [arXiv: 1211.6096] [INSPIRE].

[7] CMS collaboration, Measurement of the $p p \rightarrow Z Z$ production cross section and constraints on anomalous triple gauge couplings in four-lepton final states at $\sqrt{s}=8$ TeV, Phys. Lett. B 740 (2015) 250 [arXiv:1406.0113] [INSPIRE].

[8] CMS collaboration, Search for $W W \gamma$ and $W Z \gamma$ production and constraints on anomalous quartic gauge couplings in pp collisions at $\sqrt{s}=8$ TeV, Phys. Rev. D 90 (2014) 032008 [arXiv:1404.4619] [INSPIRE].

[9] S.H.H. Tye and S.S.C. Wong, Bloch wave function for the periodic sphaleron potential and unsuppressed baryon and lepton number violating processes, Phys. Rev. D 92 (2015) 045005 [arXiv: 1505.03690] [INSPIRE].

[10] V.A. Kuzmin, V.A. Rubakov and M.E. Shaposhnikov, On the anomalous electroweak baryon number nonconservation in the early universe, Phys. Lett. B 155 (1985) 36 [INSPIRE].

[11] M. Fukugita and T. Yanagida, Baryogenesis without grand unification, Phys. Lett. B 174 (1986) 45 [INSPIRE].

[12] V.A. Rubakov and M.E. Shaposhnikov, Electroweak baryon number nonconservation in the early universe and in high-energy collisions, Usp. Fiz. Nauk 166 (1996) 493 [Phys. Usp. 39 (1996) 461] [hep-ph/9603208] [INSPIRE].

[13] A.G. Cohen, D.B. Kaplan and A.E. Nelson, Progress in electroweak baryogenesis, Ann. Rev. Nucl. Part. Sci. 43 (1993) 27 [hep-ph/9302210] [INSPIRE].

[14] W. Buchmüller, P. Di Bari and M. Plümacher, Leptogenesis for pedestrians, Annals Phys. 315 (2005) 305 [hep-ph/0401240] [INSPIRE].

[15] L.D. McLerran, A.I. Vainshtein and M.B. Voloshin, Electroweak interactions become strong at energy above approximately 10 TeV, Phys. Rev. D 42 (1990) 171 [INSPIRE].

[16] V.V. Khoze and A. Ringwald, Total cross-section for anomalous fermion number violation via dispersion relation, Nucl. Phys. B 355 (1991) 351 [INSPIRE].

[17] A. Ringwald, An upper bound on the total cross-section for electroweak baryon number violation, JHEP 10 (2003) 008 [hep-ph/0307034] [INSPIRE]. 
[18] T. Banks, G.R. Farrar, M. Dine, D. Karabali and B. Sakita, Weak interactions are weak at high-energies, Nucl. Phys. B 347 (1990) 581 [InSPIRE].

[19] J. Ellis and K. Sakurai, Search for sphalerons in proton-proton collisions, JHEP 04 (2016) 086 [arXiv: 1601.03654 ] [INSPIRE].

[20] M.J. Gibbs and B.R. Webber, HERBVI: a program for simulation of baryon and lepton number violating processes, Comput. Phys. Commun. 90 (1995) 369 [hep-ph/9504232] [INSPIRE].

[21] A. Ringwald, Electroweak instantons/sphalerons at VLHC?, Phys. Lett. B 555 (2003) 227 [hep-ph/0212099] [INSPIRE].

[22] Pierre Auger collaboration, J.R.T. de Mello Neto, Measurements of cosmic rays at the highest energies with the Pierre Auger Observatory, Adv. Space Res. 53 (2014) 1476 [arXiv: 1309.1249] [INSPIRE].

[23] G. Brooijmans, P. Schichtel and M. Spannowsky, Cosmic ray air showers from sphalerons, arXiv: 1602.00647 [INSPIRE].

[24] D.A. Morris and A. Ringwald, Cosmic ray signatures of multi-W processes, Astropart. Phys. 2 (1994) 43 [hep-ph/9308269] [INSPIRE].

[25] A. Ringwald and H. Tu, Collider versus cosmic ray sensitivity to black hole production, Phys. Lett. B 525 (2002) 135 [hep-ph/0111042] [INSPIRE].

[26] Z. Fodor, S.D. Katz, A. Ringwald and H. Tu, Electroweak instantons as a solution to the ultrahigh-energy cosmic ray puzzle, Phys. Lett. B 561 (2003) 191 [hep-ph/0303080] [INSPIRE].

[27] T. Han and D. Hooper, Effects of electroweak instantons in high-energy neutrino telescopes, Phys. Lett. B 582 (2004) 21 [hep-ph/0307120] [INSPIRE].

[28] J.I. Illana, M. Masip and D. Meloni, Cosmogenic neutrinos and signals of TeV gravity in air showers and neutrino telescopes, Phys. Rev. Lett. 93 (2004) 151102 [hep-ph/0402279] [INSPIRE].

[29] ICECube collaboration, J. Ahrens et al., Sensitivity of the IceCube detector to astrophysical sources of high energy muon neutrinos, Astropart. Phys. 20 (2004) 507 [astro-ph/0305196] [INSPIRE].

[30] ICECuBE collaboration, IceCube preliminary design document, https://icecube.wisc.edu/icecube/static/reports/IceCubeDesignDoc.pdf, (2001).

[31] KM3NeT collaboration, R. Coniglione, The KM3NeT neutrino telescope, J. Phys. Conf. Ser. 632 (2015) 012002 [INSPIRE].

[32] V.S. Berezinsky and G.T. Zatsepin, Cosmic rays at ultrahigh-energies (neutrino?), Phys. Lett. B 28 (1969) 423 [INSPIRE].

[33] K. Greisen, End to the cosmic ray spectrum?, Phys. Rev. Lett. 16 (1966) 748 [INSPIRE].

[34] G.T. Zatsepin and V.A. Kuzmin, Upper limit of the spectrum of cosmic rays, JETP Lett. 4 (1966) 78 [Pisma Zh. Eksp. Teor. Fiz. 4 (1966) 114] [InSPIRE].

[35] N.S. Manton, Topology in the Weinberg-Salam theory, Phys. Rev. D 28 (1983) 2019 [INSPIRE].

[36] T. Akiba, H. Kikuchi and T. Yanagida, Static minimum energy path from a vacuum to a sphaleron in the Weinberg-Salam model, Phys. Rev. D 38 (1988) 1937 [InSPIRE]. 
[37] M. Ahlers, L.A. Anchordoqui, M.C. Gonzalez-Garcia, F. Halzen and S. Sarkar, GZK neutrinos after the Fermi-LAT diffuse photon flux measurement, Astropart. Phys. 34 (2010) 106 [arXiv: 1005.2620] [INSPIRE].

[38] IceCube collaboration, M.G. Aartsen et al., Probing the origin of cosmic rays with extremely high energy neutrinos using the IceCube observatory, Phys. Rev. D 88 (2013) 112008 [arXiv: 1310.5477] [INSPIRE].

[39] ICECube collaboration, T. Montaruli, Neutrino physics and astrophysics with IceCube, in Cosmic Ray International Seminar: the status and the future of the UHE Cosmic Ray Physics in the post LHC era (CRIS 2015), Gallipoli Italy September 14-16 2015 [arXiv: 1512.07978] [INSPIRE].

[40] ATLAS collaboration, Search for strong gravity in multijet final states produced in $p p$ collisions at $\sqrt{s}=13 \mathrm{TeV}$ using the ATLAS detector at the LHC, JHEP 03 (2016) 026 [arXiv:1512.02586] [INSPIRE]. 